\title{
Nuevas estrategias de mediación parental en el uso de las redes sociales por adolescentes
}

\section{New strategies of parental mediation in the use of social networks by adolescents}

\author{
María-Cruz López-De-Ayala; Esther Martínez-Pastor; Beatriz Catalina-García
}

Cómo citar este artículo:

López-De-Ayala, María-Cruz; Martínez-Pastor, Esther; Catalina-García, Beatriz (2019). “Nuevas estrategias de mediación parental en el uso de las redes sociales por adolescentes". El profesional de la información, v. 28, n. 5, e280523.

https://doi.org/10.3145/epi.2019.sep.23

Artículo recibido el 13-03-2019 Aceptación definitiva: 20-05-2019

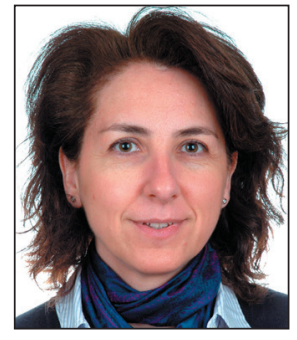

María-Cruz López-De-Ayala $\triangle$ http://orcid.org/0000-0002-6989-866X

Universidad Rey Juan Carlos Departamento de Ciencias de la Comunicación y Sociología Camino del Molino, $\mathrm{s} / \mathrm{n}$. 29943 Fuenlabrada (Madrid), España mariacruz.lopezdeayala@urjc.es

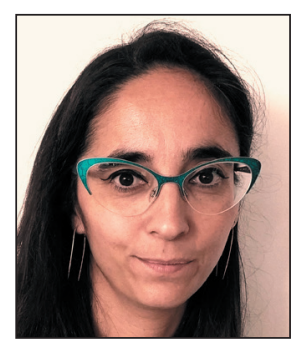

Esther Martínez-Pastor http://orcid.org/0000-0002-2861-750X

Universidad Rey Juan Carlos Departamento de Ciencias de la Comunicación y Sociología Camino del Molino s/n. 29943 Fuenlabrada (Madrid), España esther.martinez.pastor@urjc.es

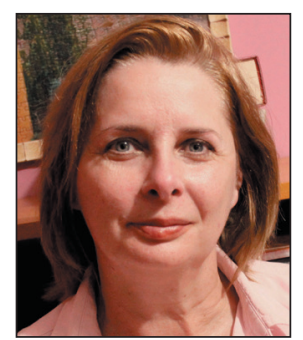

\section{Beatriz Catalina-García} https://orcid.org/0000-0003-0464-3225

Universidad Rey Juan Carlos Departamento de Ciencias de la Comunicación y Sociología Camino del Molino s/n. 29943 Fuenlabrada (Madrid), España beatriz.catalina@urjc.es

\section{Resumen}

La diversidad, complejidad y carácter cambiante de las prácticas de mediación parental dificulta que la comunidad científica establezca una tipología consensuada de estrategias de intervención del uso online de los menores. Partiendo de veintiséis entrevistas en profundidad a progenitores de adolescentes (9-17 años) de la Comunidad de Madrid, el objetivo de este trabajo es actualizar las prácticas de los padres para mediar el uso de internet de sus hijos, poniendo especial atención en las estrategias emergentes vinculadas con los dispositivos móviles y redes sociales. Entre los resultados destacan: la preferencia por un modelo de supervisión a distancia, o deferencia, en el que los padres prefieren limitar su intervención confiando en que sus hijos harán un uso online responsable; y la identificación de una nueva práctica consistente en usos conjuntos fomentados por los padres para generar experiencias positivas compartidas que favorezcan el aprendizaje del menor.

\section{Palabras clave}

Mediación parental; Adolescentes; Uso de internet; Dispositivos móviles; Smartphones; Redes sociales; Deferencia; Aprendizaje participativo.

Financiación

Esta investigación ha sido parcialmente financiada por el Gobierno de la Comunidad de Madrid bajo el proyecto Forte-CM (S2018/TCS-4314) y por el proyecto Madrid (TIN2017-88557-R), financiado por el Ministerio de Economía y Empresa del Gobierno de España. 


\begin{abstract}
The diversity, complexity and changing nature of parental mediation practices for adolescent online use contribute to the failure to achieve a consensual typology among scientific community. Over twenty-six interviews with parents of adolescent and preadolescent children (from 9 to 17 years) of the Community of Madrid, this work aims to update the types of intervention of parents to mediate the use of their children's Internet, paying special attention to emerging strategies linked to the extension of mobile devices and social networks. The results point to: the preference for a model of remote supervision, or deference, in which parents prefer to limit their intervention, trusting that their children will be responsible with their use of the Internet; model that reconcile well with respect for the privacy of adolescents. A new practice consisting of co-use promoted by parents to generate shared positive experiences that foster the child's learning is highlighted.
\end{abstract}

\title{
Keywords
}

Parental mediation; Adolescents; Children; Young; Teens; Teenagers; Internet use; Mobile devices; Smartphones; Social networks; Deference; Participatory learning.

\section{Introducción}

Los padres están ampliando la gama de estrategias de mediación en sus intentos de aumentar las oportunidades de actividades online de sus hijos mientras reducen sus riesgos (Livingstone et al., 2017). Diversos estudios destacan la creciente intervención parental en la relación que los niños españoles mantienen con internet, con más reglas establecidas y un incremento de la mediación activa de seguridad y de peticiones de información sobre experiencias online incómodas o sospechosas (López-De-Ayala; Ponte, 2016; Garmendia-Larrañaga et al., 2016; Martínez-Fernández; Casado-Del-Río, 2018). Aunque los motivos de esta progresiva mediación pueden deberse a una mayor conciencia y preocupación sobre los riesgos de las actividades online, merece la pena tener en cuenta otros aspectos.

La potencial disponibilidad de tecnologías móviles en cualquier momento y lugar promueve un uso online más intensivo que hace sospechar que los menores están más expuestos a riesgos digitales (Mascheroni; Ólafsson, 2014). No obstante, a medida que internet se vuelve más personal y móvil, los padres tienen más dificultades para saber qué hacen sus hijos online (Clark, 2011) y regular sus usos.

La difusión de tabletas y smartphones también promueve el acceso a sistemas de mensajería instantánea como WhatsApp (Holloway; Green; Love, 2014), con un notable éxito en España (Fundación Telefónica, 2017). Paralelamente, los padres muestran una mayor concienciación sobre el peligro de publicar imágenes y videos en redes sociales o en WhatsApp (Torrecillas-Lacave; Vázquez-Barrio; Monteagudo-Barandalla, 2017), lo que podría conllevar nuevas medidas de control. Además las estrategias de mediación pueden verse afectadas por la ampliación de los conocimientos y habilidades online paternas, que derivan de un mayor uso de estos medios, y de los consejos de instituciones orientadas a proteger a los menores en la Red.

Como el uso de la tecnología digital ocurre principalmente en la familia, los padres juegan un papel crucial en su adopción y en el desarrollo de la alfabetización digital (Livingstone, 2007; Sánchez-Valle; De-Frutos-Torres; Vázquez-Barrio, 2017), al mismo tiempo que vigilan el bienestar psicosocial de sus hijos y aspiran a que se conviertan en adultos autónomos y responsables. Al incrementarse las expectativas de los padres relacionadas con el aprendizaje, la comunicación y la participación online (Martínez-Fernández; Casado-Del-Río, 2018), aumenta el interés por conocer en qué medida los padres enfocan sus prácticas de mediación online al aprendizaje en el ámbito familiar. Todo ello hace necesaria una continua actualización del conocimiento sobre el tema.

\subsection{Estado de la cuestión}

Los padres implementan diversas prácticas de atención y seguimiento de las actividades y contactos online de los jóvenes (Dishion; McMahon, 1998). Lwin, Stanaland y Miyazaki (2008) diferenciaron dos estrategias básicas de mediación en internet:

- activa: los padres están cerca de sus hijos cuando navegan, hablan con ellos sobre el uso de internet o revisan la pantalla del ordenador;

- restrictiva: se busca limitar el acceso a ciertos contenidos o el tiempo en la Red. 
Livingstone et al. (2011) distinguen entre mediación restrictiva y técnica, e incorporan el monitoreo o seguimiento de las actividades, contactos y conversaciones online de los hijos "a posteriori". Asimismo, diferencian entre:

- mediación activa de uso: que incluye hablar sobre contenido y actividades online, sentarse cerca mientras los niños navegan y compartir sus experiencias;

- mediación activa de seguridad: referida a la discusión paterno-filial para mejorar el uso seguro y responsable.

Esta última a menudo se utiliza, conjuntamente con el monitoreo online, cuando el niño ya ha experimentado una situación desagradable (Duerager; Livingstone, 2012), siendo la mediación activa y restrictiva la que prefieren los padres europeos (Livingstone et al., 2011; Garmendia-Larrañaga et al., 2013).

Recientemente, Symons et al. (2017) encontraron que la estrategia más importante para padres e hijos (13-18 años) era la "restricción de interacción" en sitios de redes sociales, seguida por:

- monitoreo,

- restricción de acceso,

- supervisión y uso conjunto como una estrategia única,

- mediación técnica, y

- mediación interpretativa.

Basándose en la bibliografía sobre la crianza, Padilla-Walker et al. (2012) proponen la deferencia como una nueva forma de mediación referida a la decisión de no intervenir y dar autonomía a los hijos, porque confían en ellos y esperan que actúen responsablemente. Esta es una práctica que aumentaría conforme los niños crecen, sin que las limitaciones de edad de la muestra utilizada (11-13 años y hasta dos años más tarde) revelen una situación clara.

Glatz, Crowe y Buchanan (2018) añaden dos nuevas categorías registradas para adolescentes:

- demandas de información sobre las actividades online con el objetivo de controlarles, difiriendo de la mediación activa;

- intentos paternos de supervisar las actividades de los niños a través de la proximidad, categorizada independientemente del co-uso.

Adicionalmente, siguiendo la propuesta de Clark (2011) de incluir el aprendizaje participativo como una nueva estrategia de mediación parental que recoge una interacción y aprendizaje online conjunto, diversos investigadores aconsejan prestar mayor atención a los aspectos educativos del uso de la tecnología digital, y no sólo a los factores de riesgo. Zaman et al. (2016) por ejemplo distinguen entre el co-uso orientado al aprendizaje temprano o al placer familiar y el dirigido a fines recreativos. Y Livingstone et al. (2017) incluyen las actividades iniciadas por los niños cuando solicitan el apoyo y la interacción con sus padres; aunque no encuentran una consistencia interna sólida de este ítem en su análisis factorial y recomiendan su revisión.

De acuerdo con lo anterior, el objetivo general de esta investigación es actualizar, refinar y profundizar en las prácticas y estrategias de mediación del uso online de los adolescentes y preadolescentes implementadas por los padres españoles en relación con los nuevos dispositivos móviles y las redes sociales, teniendo en cuenta la perspectiva dinámica y relacional de estos procesos. Los objetivos secundarios son:

- Identificar las prácticas paternas de mediación de la relación de sus hijos con internet y especialmente con los dispositivos móviles (smartphones y tabletas).

- Determinar si las estrategias de deferencia (Padilla-Walker et al., 2012) y aprendizaje participativo (Clark, 2011) están presentes entre las implementadas por los padres españoles con sus hijos y desvelar cómo se aplican.

- Establecer la relación y sinergias que los padres buscan en la combinación de diferentes prácticas.

- Profundizar en cómo los padres aplican estas prácticas según las circunstancias particulares de cada familia.

\section{Material y métodos}

Para tipificar las prácticas de intervención parental en el uso online de adolescentes y preadolescentes, se realizaron entrevistas en profundidad a veintiséis padres (20 familias) de niños entre 9 y 16 años con acceso a tabletas y/o smartphones y residentes en la Comunidad de Madrid.

El objetivo es acercarnos a las experiencias vividas por los padres y a su interpretación de las situaciones de mediación de la relación de sus hijos con los medios digitales. Se busca una mejor identificación de la diversidad y combinación de estrategias y prácticas que están escasamente recogidas en la bibliografía sobre el tema, incorporando las condiciones de su aplicación.

Los entrevistados fueron reclutados buscando la máxima variación, con el objetivo de que cada familia aportase elementos de contraste al conjunto de la muestra. Las familias seleccionadas vivían en la capital de Madrid y en varios municipios de su área metropolitana. Para su reclutamiento se utilizó la técnica de bola de nieve en base a las redes sociales de los investigadores, y hasta que se alcanzó el nivel de saturación a partir del cual no se advertían nuevas aportaciones. Las edades de los padres variaban entre 33 y 52 años. El nivel de estudios varió desde educación primaria hasta estudios de postgrado, incluyendo graduado escolar, formación profesional, bachillerato y estudios universitarios. La mayor parte de las familias eran nucleares, dos monoparentales y dos reconstituidas, con niveles de ingresos muy variados. Las 
entrevistas se desarrollaron entre junio y septiembre de 2017 en las casas de los entrevistados. Cuando fue posible se entrevistó a ambos padres (6), pero en la mayoría de los casos sólo fue posible entrevistar al padre (4) o la madre (10).

Tabla 1. Características descriptivas de la muestra

\begin{tabular}{|c|c|c|c|c|c|c|c|c|}
\hline & $\begin{array}{c}\text { Rol } \\
\text { parental }\end{array}$ & $\begin{array}{l}\text { Tipo de } \\
\text { familia }\end{array}$ & Edad & Nivel de estudios & Profesión & Edad de hijos & Nacionalidad & $\begin{array}{l}\text { Rango de } \\
\text { ingresos }\end{array}$ \\
\hline F1 & Madre & Nuclear & $\begin{array}{l}P: 45 \\
M: 45\end{array}$ & $\begin{array}{l}\text { P: EGB } \\
\text { M: FP }\end{array}$ & $\begin{array}{l}\text { P: Desempleo } \\
\text { M: Asistente de } \\
\text { comedor }\end{array}$ & Hijos: 12/17 & Española & $<600$ \\
\hline $\mathbf{F} 2$ & $\begin{array}{l}\text { Padre } \\
\text { Madre }\end{array}$ & Nuclear & $\begin{array}{l}P: 47 \\
M: 45\end{array}$ & $\begin{array}{l}\text { P: FP } \\
\text { M: Universit. }\end{array}$ & $\begin{array}{l}\text { P: Oper. gráfico } \\
\text { M: ama de casa }\end{array}$ & Hijo: 13 & Española & $900-1.200$ \\
\hline $\mathbf{F 3}$ & Madre & Nuclear & $\begin{array}{l}P: 44 \\
M: 44\end{array}$ & $\begin{array}{l}\text { P: Universit. } \\
\text { M: FP }\end{array}$ & $\begin{array}{l}\mathrm{P}: \text { Veterinario } \\
\text { M: Administrador }\end{array}$ & Hijas: 10/12 & Colombiana & $1.200-1.800$ \\
\hline $\mathbf{F 4}$ & $\begin{array}{l}\text { Padre } \\
\text { Madre }\end{array}$ & Nuclear & $\begin{array}{l}P: 50 \\
M: 48\end{array}$ & $\begin{array}{l}\text { P: FPI } \\
\text { M: Primaria }\end{array}$ & $\begin{array}{l}\text { P: Operador } \\
\text { M: asistenta }\end{array}$ & Hijas: 9/14 & Española & $1.800-2.400$ \\
\hline $\mathbf{F 5}$ & $\begin{array}{l}\text { Padre } \\
\text { Madre }\end{array}$ & Nuclear & $\begin{array}{l}P: 47 \\
M: 47\end{array}$ & $\begin{array}{l}\text { P: Primaria } \\
\text { M: EGB }\end{array}$ & $\begin{array}{l}\text { F: Mecánico } \\
\text { M: Asistenta (may.) }\end{array}$ & Hija: 13 & Española & $1.800-2.400$ \\
\hline F6 & Madre & Nuclear & $\begin{array}{l}P: 43 \\
M: 46\end{array}$ & $\begin{array}{l}\text { P: Bachiller } \\
\text { M: Diplomado }\end{array}$ & $\begin{array}{l}\text { P: Informático } \\
\text { M: Auxiliar de biblio- } \\
\text { teca }\end{array}$ & $\begin{array}{l}\text { Hija: } 8 \\
\text { Hijo: } 11\end{array}$ & Española & $2.400-3.000$ \\
\hline F7 & $\begin{array}{l}\text { Padre } \\
\text { Madre }\end{array}$ & Nuclear & $\begin{array}{l}P: 44 \\
M: 41\end{array}$ & $\begin{array}{l}\text { F: Universit. } \\
\text { M: Bachiller }\end{array}$ & $\begin{array}{l}\text { P: Informático } \\
\text { M: Profesora educ. } \\
\text { especial }\end{array}$ & $\begin{array}{l}\text { Hijo: } 11 \\
\text { Hijas: } 7 / 13\end{array}$ & Española & $2.400-3.000$ \\
\hline F8 & $\begin{array}{l}\text { Padre } \\
\text { Madre }\end{array}$ & Recompuesta & $\begin{array}{l}P: 33 \\
M: 35\end{array}$ & $\begin{array}{l}\text { P: Secundaria } \\
\text { M: Primaria }\end{array}$ & $\begin{array}{l}\text { P: Gestión logística } \\
\text { M: Gestora de tienda }\end{array}$ & Hijo: 14 & Española & $3.000-4.500$ \\
\hline F9 & Padre & Nuclear & $\begin{array}{l}P: 42 \\
M: 35\end{array}$ & $\begin{array}{l}\text { P: Universit. } \\
\text { M: Universit. }\end{array}$ & $\begin{array}{l}\text { P: Banca } \\
\text { M: Ama de casa }\end{array}$ & $\begin{array}{l}\text { Hijo: } 15 / 8 / 1 \\
\text { Hijas: } 13 / 7\end{array}$ & $\begin{array}{l}\text { Española } \\
\text { Italiana }\end{array}$ & $>6.000$ \\
\hline F10 & $\begin{array}{l}\text { Padre } \\
\text { Madre }\end{array}$ & Recompuesta & $\begin{array}{l}\text { P: } 39 \\
\text { M: } 44\end{array}$ & $\begin{array}{l}\text { P: Ingeniero } \\
\text { M: FP }\end{array}$ & $\begin{array}{l}\text { P: Técnico de mante- } \\
\text { nimiento } \\
\text { M: Aux. vuelo }\end{array}$ & $\begin{array}{l}\text { Hijos: } 13 / 8 \\
\text { Hija: } 10\end{array}$ & Española & $1.800-2.400$ \\
\hline F11 & Madre & Nuclear & $\begin{array}{l}P: 50 \\
M: 46\end{array}$ & $\begin{array}{l}\text { P: Posgrado } \\
\text { M: Posgrado }\end{array}$ & $\begin{array}{l}\text { P: Banca } \\
\text { M: Sist. Informáticos }\end{array}$ & $\begin{array}{l}\text { Hijo: } 14 \\
\text { Hija: } 16\end{array}$ & Española & $2.400-3.000$ \\
\hline F12 & Madre & Monoparental & M: 40 & M: Master & M:Traductora & $\begin{array}{l}\text { Hija: } 12 \\
\text { Hijo: } 10\end{array}$ & Española & $1.200-1.800$ \\
\hline F13 & Padre & Nuclear & $\begin{array}{l}\text { P:52 } \\
\text { M: } 52\end{array}$ & $\begin{array}{l}\text { P: Técnico superior } \\
\text { M: Técnico superior }\end{array}$ & $\begin{array}{l}\text { P: Director de cen- } \\
\text { tros comerciales } \\
\text { M: Administrativa }\end{array}$ & Hijo: 11/14/19 & Española & $2.400-3.000$ \\
\hline F14 & Madre & Monoparental & $M: 46$ & M: Posgrado & M: Músico & $\begin{array}{l}\text { Hijos mellizos: } 13 \\
\text { Hija: } 15\end{array}$ & Española & $2.400-3.000$ \\
\hline F15 & Madre & Nuclear & $\begin{array}{l}P: 44 \\
M: 42\end{array}$ & $\begin{array}{l}\text { P: Posgrado } \\
\text { M: Universit. }\end{array}$ & $\begin{array}{l}\text { P: Profesor univer- } \\
\text { sitario } \\
\text { M: Artesana }\end{array}$ & Hijos: 7/10/13 & Española & $3.000-4.500$ \\
\hline F16 & Madre & Nuclear & $\begin{array}{l}P: 42 \\
M: 44\end{array}$ & $\begin{array}{l}\text { P: Doctor } \\
\text { M: Universit. }\end{array}$ & $\begin{array}{l}\text { P: Dir. creativa } \\
\text { M: Dir. financiero }\end{array}$ & $\begin{array}{l}\text { Hijas: } 16 / 10 \\
\text { Hijo: } 15\end{array}$ & Española & $>6.000$ \\
\hline F17 & Madre & Nuclear & $\begin{array}{l}P: 45 \\
M: 45\end{array}$ & $\begin{array}{l}\text { P: Universit. } \\
\text { M: Universit. }\end{array}$ & $\begin{array}{l}\text { P: Economista } \\
\text { M: Bibliotecaria }\end{array}$ & Hijas: 10/14 & Española & $1.200-1.800$ \\
\hline F18 & Madre & Nuclear & $\begin{array}{l}P: 40 \\
M: 38\end{array}$ & $\begin{array}{l}\text { P: Bachiller } \\
\text { M: Universit. }\end{array}$ & $\begin{array}{l}\text { P: Operario } \\
\text { M: Maestra }\end{array}$ & Hijas: 3/8/12 & Española & $1.200-1.800$ \\
\hline F19 & Padre & Nuclear & $\begin{array}{l}P: 47 \\
M: 52\end{array}$ & $\begin{array}{l}\text { P: Universit. } \\
\text { M: Universit. }\end{array}$ & $\begin{array}{l}\text { P: Periodista } \\
\text { M: Socióloga }\end{array}$ & Hija: 15 & Española & $4.500-6.000$ \\
\hline F20 & Padre & Nuclear & $\begin{array}{l}P: 46 \\
M: 46\end{array}$ & $\begin{array}{l}\text { P: Universit. } \\
\text { M: Bachiller }\end{array}$ & $\begin{array}{l}\text { P: Periodista } \\
\text { M: Secretaria de } \\
\text { dirección }\end{array}$ & Hijo: 10 & Española & $3.000-4.500$ \\
\hline
\end{tabular}

Los autores de este artículo realizaron las entrevistas, cuya duración osciló entre 33 y 78 minutos. Previamente los participantes fueron informados de los objetivos del estudio, sus derechos y la confidencialidad. Las entrevistas fueron semi-estructuradas, utilizando preguntas amplias y orientativas, fueron grabadas y los datos transcritos. Estos se etiquetaron, organizaron y analizaron por diferentes temas y categorías según las preguntas de la investigación, y de forma manual.

Las categorías para la codificación se establecieron sobre la revisión de la bibliografía previa y un primer examen de la transcripción de las entrevistas, registrando la diversidad práctica recogida en cada tipo de mediación, así como posibles opciones no recogidas en la bibliografía anterior. Se incluyó: 
- mediación restrictiva, que incluye normas de restricción de acceso a dispositivos, contenidos y actividades (Symons et al., 2017), y restricción técnica (Livingstone et al., 2011);

- supervisión, que integra también supervisión a distancia o deferencia (Padilla-Walker et al., 2012);

- mediación activa, incluyendo mediación activa de riesgos, mediación activa de uso (Livingstone et al., 2001), co-uso y aprendizaje colectivo (Clark, 2011; Zaman et al., 2016; Livingstone et al., 2017).

Al ejemplificar con extractos de entrevistas, y para preservar el anonimato de los entrevistados, se han utilizado las abreviaturas $\mathrm{M}$ y $\mathrm{P}$ para referirse a la madre y al padre, respectivamente, seguidos por la letra $\mathrm{F}$ y el número de la entrevista que identifica a cada familia. Con el fin de facilitar la lectura, únicamente se recogen las características de los entrevistados cuando se considera significativo para la comprensión e interpretación del análisis.

\section{Resultados}

Los padres entienden que su obligación es controlar el uso online de sus hijos, en tanto que éstos no sean capaces de gestionar sus actividades y demostrar su madurez. El objetivo a largo plazo es que sean capaces de autorregularse y de "reaccionar" (M_F4, M_F7) y para ello los padres deben transmitir unos valores

"establecidos de manera convencional, [que] son los mismos que aplican en los dispositivos electrónicos" (M_F16);

porque

"hay los mismos peligros y no puedes acotar todo" (M_F11);

"es mejor prepararles para lo que se enfrentan antes que decir no, porque al final van a ir a clase y se lo va a enseñar el de al lado en el móvil” (M_F6).

En general, los progenitores de ambos sexos se muestran muy moderados en sus formas de control, valorándose negativamente tanto la prohibición como la permisividad absoluta, que asocian con el desconocimiento tecnológico que genera más temor o menos conciencia de los riesgos. Algunos padres y madres aceptan ser más severos, y lo justifican porque

"mis hijas me engañan" y "es mi obligación velar por ellas, no quiero cargar con esa culpa” (M_F3);

"[como padre] tienes una responsabilidad" (P_F10).

\subsection{Mediación restrictiva}

La mayoría de los entrevistados se muestran contrarios a un modelo educativo autoritario y admite que no tiene normas concretas o que éstas son muy laxas, manifestándose más como preferencias o consejos, y decantándose por la mediación activa y la supervisión a distancia:

"Más que prohibir, es un poco saber qué es lo que están haciendo" (P_F9).

Con el objetivo de alcanzar una autoridad negociada, se alude a la necesidad de argumentar y convencerles de la necesidad de tener normas o de su aplicación. Aunque declaran que si vieran que sus hijos hacen un uso sospechoso tomarían medidas, como prohibir o restringir el uso.

\subsubsection{Normas de restricción de acceso a dispositivos}

Numerosos padres expresan su deseo de retrasar el acceso al smartphone y a las redes sociales; pero se percibe como algo inevitable, por la presión de los amigos y porque

"negarse a algo que lo van a tener todos es muy difícil" (M_F6),

ya que provocaría que sus hijos se vieran marginados del grupo y de la sociedad.

Paralelamente muchos de ellos inciden en la necesidad de aprender a usar la tecnología desde edades tempranas,

"para evitar posibles problemas" (P_F7)

y para que

"sean autosuficientes en el manejo de las tecnologías" (P_F20).

Pero sólo un pequeño grupo, varones principalmente, piensan que deben enseñarles ellos mismos su uso lo antes posible:

"Su padre dice que esto hay que usarlo, y cuanto antes nosotros les enseñemos a usarlo, mejor" (M_F6);

"Y fue su padre el que pensó: ahora tiene 12 años y todavía nos escucha. Es mejor que vaya aprendiendo con nosotros" (M_F12).

Entre sus obligaciones los padres incluyen el control para limitar el consumo excesivo en los dispositivos móviles. Sin embargo, más que establecer una norma clara y bien definida, evalúan de forma que puede parecer arbitraria si sus hijos llevan demasiado tiempo y les piden que dejen de usarlo, se lo quitan o esconden. La aplicación de la norma es flexible y se ajusta a las circunstancias: si se están entreteniendo (F9) o no tienen otra cosa que hacer (F4). 
Además de cumplir con sus obligaciones escolares, otras restricciones vinculadas al contexto de uso son: la hora de la comida, reuniones familiares o conversaciones sociales, antes de ir a dormir o fuera de casa. Una medida para limitar el uso online de los preadolescentes que ya tienen teléfono móvil consiste en permitirles el acceso únicamente con la wifi familiar. Los más mayores (estudiantes de ESO) ya cuentan con datos propios, y deben graduar su uso hasta la próxima recarga.

Resulta común la limitación del acceso a dispositivos móviles como castigo por haber incumplido normas de tiempo o hacer un uso inadecuado; no obstante se cuestiona la idoneidad de su aplicación entre los adolescentes porque

"corté una parte de sociabilidad con sus amigos, y le hicimos un daño" (P_F9, hijo 15);

"al final esto hace que estés conectado con todo el mundo, que no se sienta solo" (M_F8, hijo único 14).

Asimismo, la madre en F5 da la razón a su hija cuando le contra argumentó:

"mamá, pero si me lo quitas ¿cómo voy a aprender si no me lo das?".

Por el contrario, la madre en F7 y el padre en F10 valoran que a sus hijos preadolescentes les resulta indiferente puesto que simplemente cambian de actividad.

\subsubsection{Normas de control de actividades o acceso a contenidos}

Las normas de prohibición más comunes son tener una cuenta abierta, subir fotografías o contenidos y hablar con extraños en redes sociales, al menos, sin supervisión paterna. Otras normas aplicadas son la prohibición de bajarse/comprar juegos, hablar con extraños en juegos multiplayer o colgar información personal online. Además los hijos deben consultarles para acceder a una nueva aplicación, red social o bajarse un juego; y otros confían en que les informarían de esa actividad, aunque no señalan normas explícitas al respecto.

\subsubsection{Restricción técnica}

Cuatro de las familias han instalado filtros para limitar especialmente los juegos violentos y contenidos pornográficos. Dos mencionan que lo hicieron pensando en los hijos más mayores "porque tienen más uso" (M_F4); F15, porque su hijo "no tiene la madurez para acceder a cierta información que no puede asimilar"; y la madre en F16, cuando supo que su hijo "se interesaba" por contenido adulto.

Las motivaciones para no usarlos son:

- desconfianza en la efectividad del sistema, y

"que te limita otras cosas que sí necesitas" (P_F10);

- no requerirlos porque saben qué están haciendo sus hijos:

"no hemos tenido ninguna dificultad" (P_F20);

"confío mucho en ellas y ya son mayores" (M_F14);

- prefieren educarles

"para que no les haga falta" (P_F7),

"los filtros, al igual que la vigilancia excesiva, son contraproducentes" (M_F11);

- no saber instalarlos.

\subsection{Supervisión}

La vigilancia adopta diferentes grados y formas:

- echar un vistazo de vez en cuando y preguntarles qué hacen;

- revisar el historial (aunque ninguno declara hacerlo);

- inspeccionar contactos y conversaciones en el WhatsApp;

- examinar publicaciones en redes sociales.

La primera requiere complementarse con medidas como situar los dispositivos en espacios colectivos o pedir a los preadolescentes, principalmente, que mantengan la puerta abierta de su habitación. Las otras resultan, incluso, más intrusivas e implica revisar los dispositivos y cuentas de los hijos, con o sin su conocimiento, lo que puede exigir utilizar herramientas técnicas de rastreo, conocer sus claves de acceso y/o estar vinculados como "amigos" en redes sociales.

En consecuencia, las obligaciones de supervisión online entran en contradicción con el derecho de los adolescentes a mantener cierta privacidad, y sitúan a los padres en una posición difícil. Para afrontar esta incompatibilidad, la mayoría da por supuesto el carácter disuasorio para los adolescentes de conocer que pueden ser supervisados, limitando sus actividades a aquellas que serán aprobadas por sus padres porque saben que "se puede buscar un problema" (M_F8); como se manifiesta en este ejemplo:
Los padres confían en que los hijos no realizan actividades de riesgo online debido a que son conscientes de que pueden ser supervisados por sus padres, pero rara vez lo hacen para no violentar su privacidad 
"Entiendo que si saben que yo tengo acceso a sus redes sociales, sus WhatsApp y demás, y ellos no tienen ningún tipo de miedo a que lo mire, tampoco dirán nada malo o exageradamente salido de tono" (P_F9).

No obstante, la madre en F5 ha sentido la necesidad de hacer valer esa capacidad, supervisado las conversaciones de su hija delante de ella; pero lo hizo "por encima", lo que demuestra que no es una situación que le haya resultado cómoda.

Por último, dos padres refieren haber realizado intervenciones demasiado intrusivas, y prefieren ocultárselas a sus hijos para no desvelar que han traicionado su privacidad y confianza. El padre en F19 permitió que el técnico que instaló el ordenador incluyera un programa para seguir la navegación de su hija, pero afirma: "es como intentar leer el diario a tu hija. Pues es una cosa muy fea"; después de verificar que no hacía un uso distinto del que se cabría esperar, lo eliminó: "porque mi confianza en xxx es enorme" (P_F19). Y la madre en F11 aprovechó que su hijo, que "aún es pequeño y no se da cuenta", se dejó su sesión de Instagram abierta para examinar su perfil.

Una forma alternativa de vigilancia entre los más jóvenes consiste en revisar los contenidos de los juegos y videos que suben los youtubers a los que siguen sus hijos, por ejemplo en F7 y F3. Y en F7, F9 y F12, prefieren explorar las redes sociales antes de que sus hijos lleguen a utilizarlas.

\subsubsection{Supervisión a distancia o deferencia}

Los padres deben moverse en un terreno complicado, lidiando por encontrar un equilibrio entre garantizar la seguridad de los hijos y respetar su derecho a la privacidad, para no dañar la confianza mutua. En general, entienden que su deber es supervisar, más o menos en la distancia, el comportamiento en la Red de sus hijos adolescentes sin violentar su privacidad:

"[hay que dejarle] ese margen de libertad y confianza" (P_F20);

"si tienes un tipo de relación de confianza con tu hijo, meterte de esa manera en su vida" (M_F12).

Pero ante las dudas que a veces surgen sobre la sinceridad de los hijos, hay "estar siempre atentos" (M_F16) a cualquier indicio que indique que hay algún problema, como malos resultados escolares, aislamiento social, "comportamiento más frágil o a la defensiva", "el rechazo al propio aparato" o "su actitud en general" (P_F13).

Por lo tanto, el modo de control dominante es la supervisión a distancia, con los padres confiando en que sus hijos actúan de forma responsable y que les revelarán, de forma más o menos espontánea, sus actividades y experiencias online peligrosas o molestas.
La confianza entre padres e hijos es un factor central que orienta la actuación de los padres en su mediación online

\subsection{Mediación activa}

Es común demandar información a los hijos sobre actividades que puedan resultar problemáticas:

"les digo que si alguna vez se encontrasen en apuros, cualquier cosa rara..." (M_F14);

"les he advertido: si alguna vez os insultan por teléfono, os amenazan o pasa algo, de inmediato me tenéis que avisar" (M_F4).

\subsubsection{Mediación activa de riesgos}

Entre las responsabilidades paternas se incluye advertir a los menores de los peligros online, previniendo usos inadecuados y conductas de riesgo. Sin embargo, más que enseñarles los riesgos y a afrontarlos, los padres se limitan a comunicárselos y recordárselos.

Todos los entrevistados admiten haber hablado en familia de los peligros de internet pero, como en el caso del padre en F13, "de manera taxativa, no. De manera indirecta". Se aprovecha "cada vez que hay un caso, que surge una noticia en televisión o que se habla de ello" (P_F13), coincidiendo con charlas en el colegio o películas que tratan sobre el tema (P_F5), porque "el conocimiento de los padres en este terreno es limitado" (P_F13).

En otros casos, el diálogo sucede cuando los menores han experimentado una situación peligrosa. Una barrera que dificulta la conversación sobre los riesgos, y específicamente sobre contenidos pornográficos, se refiere a la incertidumbre sobre la edad apropiada para tratar estas cuestiones. Los padres temen "abrirles a determinados temas" (M_F10) antes de que estén preparados para abordarlos, y cuando piensan seriamente en hacerlo, perciben que ya saben más que ellos mismos. Por lo tanto, las charlas a menudo suceden cuando conocen que sus hijos han accedido a contenidos adultos; pero entonces, los padres se sienten incómodos porque piensan que forma parte de la vida privada de sus hijos. Por ejemplo, los padres en F9 y F10 no han hablado con sus hijos por esta razón; y la madre en F10 señala:

“Entonces yo le cogí un día paseando y le dije: 'tenemos que hablar tú y yo de esto, porque esto...' Se empezó a poner rojo y yo le dije: 'ten cuidado, ten cuidado con lo que haces y no haces. Yo puedo entender que tú te estés 
descubriendo pero...' Entonces, claro, como también le dio muchísima vergüenza, se puso rojo y eso, lo dejé ahí. [...] Es que mi niño, todavía era mi niño. No me lo imaginaba".

\subsubsection{Mediación activa de uso}

Diferentes ejemplos muestran que los padres hablan a sus hijos sobre los contenidos y actividades online, comparten actividades y les exponen sus propias experiencias online; no obstante, la frontera que separa la mediación activa de uso, relativa a aspectos técnicos del uso, y de riesgo es difusa, y ambas estrategias se solapan.

\subsubsection{Co-uso y aprendizaje colaborativo}

Encontramos diferentes situaciones de tiempo compartido online entre padres e hijos, orientadas al ocio familiar, aprendizaje o mediación activa de riesgos. Habitualmente son los hijos quienes inician el co-uso de entretenimiento en familia, mostrando a sus padres contenidos curiosos o divertidos que han encontrado en YouTube; además, los padres en F9 y F7 visualizan partidos de fútbol y juegan con sus hijos varones. Los menores también muestran a sus padres sus actividades o logros online, aunque éstos no siempre tienen tiempo y manifiestan desinterés, limitando la interacción al comprobar que no hay nada peligroso.

Algunos padres y madres (M_F6, M_F10, P_F9, y P y M_F5) mencionan situaciones de aprendizaje vertical ascendente. Además, se relatan usos conjuntos iniciados por los padres que están orientados a generar experiencias positivas compartidas que favorezcan el aprendizaje del menor. Algunos padres enseñan a sus hijos el uso de las redes sociales "porque tienen que ser competentes" (M_F7), con el objetivo de que aprendan un buen uso; y, paralelamente, les muestran ejemplos de malos usos y sus respuestas ante situaciones conflictivas. A modo de ejemplo, la madre en F12 enseña a su hija las

"cosas que me pasan a mí en Facebook"... "A veces, de discusiones que he tenido, le voy explicando cómo lo hago".

El padre en F19 inició a su hija en el funcionamiento de Twitter cuando ésta participó en una campaña de Greenpeace en esta plataforma. Y el padre en F20 manifiesta:

"Le he explicado el funcionamiento de Facebook o Twitter para que tenga nociones. Siempre aprovecho para decir 'mira qué mal uso ha hecho este señor de la Red. ¿Qué te parece? Mal porque lo puede ver no sé quién'. Para que, poco a poco, vaya interiorizando una serie de pautas".

Cabe destacar que todos los padres que participan en este aprendizaje guiado manifestaban una posición más favorable al uso temprano de las tecnologías, dirigido a orientar unas prácticas saludables en sus hijos.

\section{Conclusiones}

Con el objetivo de identificar nuevas prácticas emergentes en la mediación parental con adolescentes y preadolescentes sobre el uso de dispositivos móviles y redes sociales, en esta investigación se han realizado entrevistas a veintiséis padres y madres residentes en la Comunidad de Madrid.

Los entrevistados se muestran contrarios, en general, a un modelo educativo autoritario y se decantan por un modelo basado en el diálogo y la confianza mutua. Sin embargo, similar a los resultados de Erickson et al. (2016), el grado de libertad y control ejercido en la práctica varía notablemente entre los progenitores, incluso en una misma familia, en relación con la percepción del nivel de madurez y de la importancia que otorgan a las demandas de privacidad de sus hijos. En general se muestran muy moderados en sus formas de control, valorándose negativamente tanto la prohibición como la permisividad absoluta.

En línea con los hallazgos de Symons et al. (2017), las reglas y restricciones más comunes son tener una cuenta abierta, subir fotografías o contenidos y hablar con extraños en redes sociales, o hacerlo sin el conocimiento de sus padres. Mientras que, en coherencia con los resultados de Martínez-Fernández y Casado-Del-Río (2018), el uso de software de filtrado no está generalizado en nuestra muestra. Aunque, a diferencia de sus resultados que apuntan a una tendencia creciente del uso de software de filtrado en España que afecta especialmente a los hogares con niños más jóvenes, la mitad de los padres que los aplicaron en nuestra muestra los instalaron pensando en los más mayores; si bien todos habían vivido experiencias negativas online.

Los padres se apoyan en el diálogo para prevenir malos usos y proteger el bienestar de los adolescentes en el mundo virtual, pero también para favorecer que aprovechen todas las oportunidades que ofrece. En coherencia con lo encontrado por López-De-Ayala y Ponte (2016) y Garmendia-Larrañaga et al. (2016), quienes ven una extensión de estas prácticas entre los padres españoles, todos los entrevistados admiten utilizar la mediación activa de riesgos, que en ocasiones se entremezcla con la mediación activa de uso, menos extendida. En otros casos, como identificaron Duerager y Livingstone (2012), el diálogo surge cuando los niños han experimentado una situación peligrosa. 
Los padres entienden que su deber es supervisar, más o menos en la distancia, el comportamiento online de sus hijos adolescentes; y únicamente se admite la imposición de medidas más intrusivas, que podrían generar conflictos, cuando se demuestra que están realizando un uso responsable. Estos datos explicarían los resultados de estudios anteriores que muestran que los padres de los adolescentes mayores son menos propensos a utilizar los métodos de mediación más intrusivos (Glatz; Crowe; Buchanan, 2018; Padilla-Walker et al., 2012; Sonck; Nikken; De-Haan, 2013). Por tanto, los padres prefieren confiar en que sus hijos actúan de forma responsable y que les revelarán, de forma más o menos espontánea, sus actividades y experiencias online peligrosas o que puedan preocuparles; modelo que se ajusta bien al modelo descrito como deferencia por Padilla-Walker et al. (2012) para niños más pequeños.

Por otra parte, la extensión de dispositivos móviles, y de la Smart TV, ha favorecido prácticas paterno-filiales conjuntas dirigidas al ocio familiar, aprendizaje o mediación activa de riesgos. Se advierten usos conjuntos en redes sociales iniciados por los padres orientados a generar experiencias compartidas positivas que favorezcan el aprendizaje del menor; actividades que son posibles porque algunos padres ya son activos usuarios de las redes sociales y entienden que sus propias experiencias pueden ser de utilidad a los menores. Si bien numerosos estudios habían detectado situaciones de aprendizaje vertical de abajo-arriba entre preadolescentes y adolescentes y sus padres (Masanet; Establés, 2018), los estudios previos no mencionan prácticas de aprendizaje vertical de arriba-abajo en relación con las redes sociales. Esta nueva práctica encajaría con el modelo de aprendizaje participativo descrito para niños más pequeños por Clark (2011), y viene a incidir en los cauces de aprendizaje informal que llevan a los adolescentes a desarrollar nuevas competencias vinculadas con la alfabetización transmedia fuera de las instituciones educativas (Scolari, 2016; Scolari et al., 2018). A este respecto debemos mencionar que, aunque tanto padres y madres insisten en la importancia de que los adolescentes desarrollen habilidades digitales para no verse marginados socialmente y facilitar su incorporación futura al mundo laboral, los discursos en los que se menciona explícitamente la conveniencia de implicarse como padres en el proceso de aprendizaje se atribuyen en mayor medida a los padres varones. De esta forma, los padres se plantean asumir activamente su papel socializador en los usos y riesgos en las plataformas de redes sociales, más allá de las advertencias y prohibiciones para la prevención de estos últimos, destacando particularmente las tácticas de afrontamiento ante ciertas circunstancias controvertidas o problemáticas.

Este trabajo evidencia que los padres intentan adaptar sus prácticas de mediación a una gran diversidad de circunstancias, y muestra la complejidad de unas prácticas que son dinámicas y cambiantes. En concreto, si bien muchas de las formas de intervención identificadas coinciden con las registradas en trabajos previos, se advierten nuevas formas de mediación que sólo habían sido identificadas con niños más pequeños, como la supervisión a distancia o deferencia y el aprendizaje participativo iniciado por los padres. Adicionalmente, los resultados de esta investigación anticipan los motivos y circunstancias que orientan la implementación de estas prácticas, y que se vinculan con un modelo de familia ideal democrática basada en la confianza mutua y el diálogo. No obstante, no hay que desdeñar las numerosas presiones que los padres reciben de diferentes instituciones para elevar su grado de intervención y control sobre los menores, así como los discursos contradictorios que les llegan de los medios en relación con su papel de mediadores del uso online de los adolescentes (Blum-Ross; Livingstone, 2018).

En cuanto a las limitaciones de este estudio, cabe señalar que la riqueza de contenidos que facilita el análisis cualitativo dificulta la identificación de estrategias simplificadas que combinen diferentes prácticas según determinadas situaciones y no permite valorar su eficacia. Por otra parte, aunque en el análisis se han intentado detectar diferencias en las respuestas recogidas entre los padres y madres, esta labor se ha visto complicada por la limitación del tamaño de la muestra, que no es representativa de la población en su conjunto, y que muestra una gran variabilidad en otras características sociodemográficas y de convivencia familiar que hacen difícil determinar si una posición detectada entre uno o varios de los sujetos de la muestra responde a un aspecto compartido por género o a cualquier otra particularidad del entrevistado. Apoyándose en los resultados de este trabajo, una investigación en proceso de diseño se propone avanzar en estos aspectos, aplicando un análisis factorial con los items identificados a una muestra representativa de adolescentes de la Comunidad de Madrid para identificar estrategias específicas de mediación familiar. Su combinación con análisis multivariables explicativos permitirá avanzar en las circunstancias que predicen la implementación de diferentes estrategias de mediación parental, incluido el género del progenitor, y su efectividad para diversos escenarios.

Para finalizar, las características específicas de nuestra muestra pueden limitar la generalización de los resultados. En particular, hemos entrevistado a padres y madres que habitan en diversas zonas urbanas de la Comunidad de Madrid, por lo tanto, debemos mostrar ciertas cautelas en la generalización de los resultados de nuestras familias al conjunto de la población española.

\section{Referencias}

Blum-Ross, Alicia; Livingstone, Sonia (2018). “The trouble with 'screen time' rules”. In: Mascheroni, Giovanna; Ponte, Cristina; Jorge, Ana (eds.). Digital parenting. The challenges for families in the digital age. Göteborg: Nordicom, pp. 179187. ISBN: 9789188855008

https://www.nordicom.gu.se/sites/default/files/kapitel-pdf/16_blum-ross_livingstone.pdf 
Clark, Lynn-Schofield (2011). "Parental mediation theory for the digital age”. Communication theory, n. 21, n. 4, pp. $323-343$. https://doi.org/10.1111/j.1468-2885.2011.01391.x

Dishion, Thomas J.; McMahon, Robert J. (1998). “Parental monitoring and the prevention of child and adolescent problem behavior: A conceptual and Empirical Formulation". Clinical child and family psychology review, v. 1, n. 1, pp. 61-75. https://doi.org/10.1023/A:1021800432380

Duerager, Andrea; Livingstone, Sonia (2012). How can parents support children's internet safety?. EU Kids Online, London, UK. ISBN: 9780853283553

http://eprints.Ise.ac.uk/42872

Erickson, Lee B.; Wisniewski, Pamela; Xu, Heng; Carroll, John M.; Rosson, Mary-Beth; Perkins, Daniel F. (2016). "The boundaries between: Parental involvement in a teen's online world". Journal of the Association for Information Sciences and Technology, v. 67, n. 6, pp. 1384-1403.

https://doi.org/10.1002/asi.23450

Fundación Telefónica (2017). La sociedad de la información en España 2016. Madrid: Ariel. ISBN: 9788408169475 https://bit.ly/2p4YQYX

Garmendia-Larrañaga, Maialen; Casado-Del-Río, Miguel-Ángel; Martínez-Fernández, Gemma; Garitaonandia-Garnacho, Carmelo (2013). "Las madres y padres, los menores e internet. Estrategias de mediación parental en España". Doxa, n. 17, pp. 99-117.

https://bit.ly/2zgehBO

Garmendia-Larrañaga, Maialen; Jiménez-Iglesias, Estefanía; Casado-Del-Río, Miguel-Ángel; Mascheroni, Giovanna (2016). Net children go mobile: Riesgos y oportunidades en internet y el uso de dispositivos móviles entre menores españoles (2010-2015). Madrid: Red.es/Universidad del País Vasco. ISBN: 9788867802883

https://www.ehu.eus/documents/10136/6317486/Txostena_Informe.pdf

Glatz, Terese; Crowe, Elizabeth; Buchanan, Christy M. (2018). "Internet-specificparentalself-efficacy:Developmental differencesand links to internet-specificmediation". Computers in human behavior, v. 84, pp. 8-17.

https://doi.org/10.1016/j.chb.2018.02.014

Holloway, Donell; Green, Lelia; Love, Carlie (2014). “'It's all about the apps': Parental mediation of pre-schoolers' digital lives". Media international Australia, v. 153, n. 1, pp. 148-156.

https://doi.org/10.1177/1329878X1415300117

Livingstone, Sonia (2007). "Strategies of parental regulation in the media-rich home". Computers in human behavior, v. 23, n. 2, pp. 920-941.

https://doi.org/10.1016/j.chb.2005.08.002

Livingstone, Sonia; Haddon, Leslie; Görzig, Anke; Ólafsson, Kjartan (2011). Risks and safety on the internet: The perspective of European children. Full findings and policy implications from the EU kids online survey of 9-16 year olds and their parents in 25 countries. LSE, London: EU Kids Online.

http://eprints.Ise.ac.uk/33731

Livingstone, Sonia; Ólafsson, Kjartan; Helsper, Ellen J.; Lupiáñez-Villanueva, Francisco; Veltri, Giuseppe A.; Folkvord, Frans (2017). "Maximizing opportunities and minimizing risks for children online: The role of digital skills in emerging strategies of parentalmediation". Journal of communication, n. 67, n. 1, pp. 82-105.

https://doi.org/10.1111/jcom.12277

López-De-Ayala, María-Cruz; Ponte, Cristina (2016). "Parental mediation in online practices of the Spanish minors: A review of empirical research". Doxa, n. 23, pp. 13-46.

https://doi.org/10.31921/doxacom.n23a1

Lwin, May O.; Stanaland, Andrea J. S.; Miyazaki, Anthony D. (2008). "Protecting children's privacy online: How parental mediation strategies affect website safeguard effectiveness". Journal of retailing, v. 84, n. 2, pp. 205-217.

https://doi.org/10.1016/j.jretai.2008.04.004

Martínez-Fernández, Gemma; Casado-Del-Río, Miguel-Ángel (2018). “La responsabilidad de las madres y los padres españoles como mediadores en el uso de internet que hacen los menores: evolución y nuevos retos". En: Jiménez-Iglesias, Estefanía; Garmendia-Larrañaga, Maialen; Casado-Del-Río, Miguel-Ángel. Entre selfies y whatsapps. Oportunidades y riesgos para la infancia y la adolescencia conectada. Barcelona: Gedisa, pp. 173-188. ISBN: 9788416919871

Masanet, Maria-José; Establés, María-José (2018). "Competencias en la prevención de riesgos, ideología y ética". En: Scolari, Carlos A. (ed.). Adolescentes, medios de comunicación y culturas colaborativas. Aprovechando las competencias transmedia de los jóvenes en el aula. Barcelona: Universidad Pompeu Fabra, pp. 74-82. ISBN: 9788409002931

http://transmedialiteracy.upf.edu/sites/default/files/files/TL_Teens_es.pdf 
Mascheroni, Giovanna; Ólafsson, Kjartan (2014). Net children go mobile: Risks and opportunities, $2^{\text {nd }}$ ed. Milano: Educatt. https://doi.org/10.13140/RG.2.1.3590.8561

Padilla-Walker, Laura M.; Coyne, Sarah M.; Fraser, Ashley M.; Dyer, W. Justin; Yorgason, Jeremy B. (2012). "Parents and adolescents growing up in the digital age: Latent growth curve analysis of proactive media monitoring". Journal of adolescence, v. 35, n. 5, pp. 1153-1165. https://doi.org/10.1016/j.adolescence.2012.03.005

Sánchez-Valle, María; De-Frutos-Torres, Belinda; Vázquez-Barrio, Tamara (2017). "La influencia de los padres en la adquisición de habilidades críticas en Internet". Comunicar, v. 25, n. 53, pp. 103-111. https://doi.org/10.3916/C53-2017-10

Scolari, Carlos A. (2016). "Alfabetismo transmedia. Estrategias de aprendizaje informal y competencias mediáticas en la nueva ecología de la comunicación". Telos, n. 103, pp. 12-23.

Scolari, Carlos A.; Masanet, Maria-José; Guerrero-Pico, Mar; Establés, María-José (2018). “Transmedia literacy in the new media ecology; Teens' transmedia skills and informal learning strategies". El profesional de la información, v. 27, n. 4, pp. 801-812.

https://doi.org/10.3145/epi.2018.jul.09

Sonck, Nathalie; Nikken, Peter; De-Haan, Jos (2013). “Determinants of internet mediation: A comparison of the reports by Dutch parents and children". Journal of children and media, v. 7, n. 1, pp. 96-113.

https://doi.org/10.1080/17482798.2012.739806

Symons, Katrie; Ponnet, Koen; Emmery, Kathleen; Walrave, Michael; Heirman, Wannes (2017). "A factorial validation of parental mediation strategies with regard to internet use". Psychologica Belgica, v. 57, n. 2, pp. 93-111.

https://doi.org/10.5334/pb.372

Torrecillas-Lacave, Teresa; Vázquez-Barrio, Tamara; Monteagudo-Barandalla, Laura (2017). “Percepción de los padres sobre el empoderamiento digital de las familias en hogares hiperconectados". El profesional de la información, v. 26, n. 1, pp. 96-104.

https://doi.org/10.3145/epi.2017.ene.10

Zaman, Bieke; Nouwen, Marije; Vanattenhoven, Jeroen; De-Ferrerre, Evelien; Van-Looy, Jan-Van (2016). "A qualitative inquiry into the contextualized parental mediation practices of young children's digital media use at home". Journal of broadcasting and electronic media, n. 60, pp. 11-22.

https://doi.org/10.1080/08838151.2015.1127240

La Fundación Biblioteca Social es una institución sin ánimo de lucro que se constituyó en el año 2014. Tiene como objetivo contribuir a compensar los desequilibrios sociales apoyando proyectos que llevan a cabo las bibliotecas públicas, dirigidos a los sectores más vulnerables de la sociedad.

\section{¿Colaboras?}

fundacionbibliotecasocial.org info@fundacionbibliotecasocial.org

3 @Biblio_Social

FundacionBibliotecasSocial

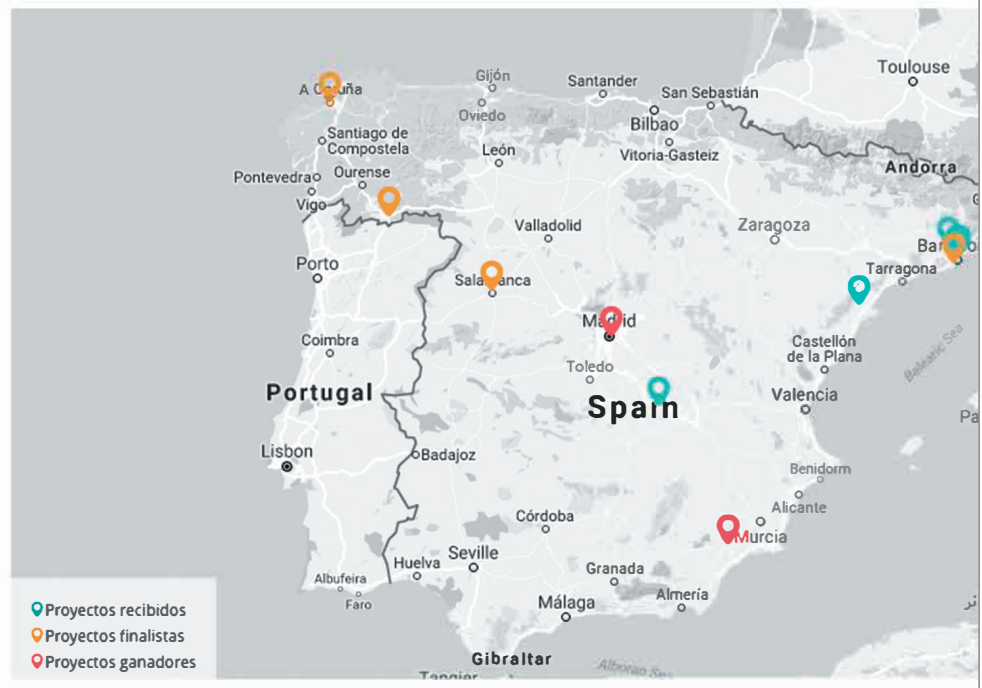

Mapa de proyectos de bibliotecas públicas para la inclusión social. 2016. 